\title{
Investigation of long non-coding RNA expression profiles in patients with post-menopausal osteoporosis by RNA sequencing
}

\author{
SHAOHAI WANG \\ Department of Obstetrics and Gynecology, Union Hospital, Tongji Medical College, \\ Huazhong University of Science and Technology, Wuhan, Hubei 430012, P.R. China
}

Received June 13, 2019; Accepted December 02, 2019

DOI: $10.3892 /$ etm.2020.8881

\begin{abstract}
The present study aimed to investigate the implication of long non-coding RNA (lncRNA) expression profiles in post-menopausal osteoporosis (PMOP). A total of 10 patients with PMOP and 10 age-matched healthy post-menopausal females as controls were consecutively enrolled. Their peripheral blood mononuclear cells were obtained and lncRNA as well as mRNA expression profiles were detected by RNA sequencing, followed by bioinformatics analyses. The lncRNA expression profiles were able to distinguish patients with PMOP from controls based on principal component analysis and heatmap analysis. In total, 254 upregulated lncRNAs and 359 downregulated lncRNAs were identified in patients with PMOP vs. controls. The top 5 upregulated lncRNAs were RP11-704M14.1,RP11-754N21.1,RP11-408E5.5,ANKRD26P3 and TPTEP1. The top 5 downregulated lncRNAs were RP11-310E22.4, RP11-326K13.4, FABP5P1, SERPINB9P1 and RPL13P2. Based on the interaction of dysregulated lncRNAs and mRNAs by RNA sequencing, functional annotations were then performed. Gene Ontology enrichment analysis revealed that the dysregulated IncRNAs were enriched in terms including apoptotic process and positive regulation of $\mathrm{NF}-\kappa \mathrm{B}$ transaction, and Kyoto Encyclopedia of Genes and Genomes analysis suggested enrichment in PMOP-associated signaling pathways, including osteoclast differentiation, tumor necrosis factor signaling pathway and mitogen-activated protein kinase signaling pathway. In addition, the regulatory network and circos graph further indicated the implication of lncRNA expression profiles in PMOP via interactions with mRNAs. In conclusion, the present study suggested that aberrant lncRNA expression is deeply involved in the pathogenesis of PMOP
\end{abstract}

Correspondence to: Dr Shaohai Wang, Department of Obstetrics and Gynecology, Union Hospital, Tongji Medical College, Huazhong University of Science and Technology, 1277 Jiefang Avenue, Wuhan, Hubei 430012, P.R. China

E-mail: shaozhuo172326073@163.com

Key words: post-menopausal osteoporosis, long non-coding RNA, expression profiles, dysregulation, signaling pathways by affecting osteoclast differentiation, inflammation and apoptotic processes.

\section{Introduction}

Osteoporosis is a common bone disorder characterized by excessive bone resorption and insufficient formation of new bone, leading to reduced bone function and increased risk of fracture (1-3). Among several subtypes of osteoporosis, post-menopausal osteoporosis (PMOP) affects $9-38 \%$ of females and accounts for a large proportion of the total osteoporotic population (4,5). PMOP is a chronic disease with high prevalence among post-menopausal females. Therefore, PMOP has been considered a major public health concern during the last decades $(6,7)$.

Long non-coding RNAs (lncRNAs) are evolutionarily conserved molecules that have been extensively studied due to their significant role in various biological processes, including signal transduction, cell growth and apoptosis (8-10). Recently, the expression levels of several specific lncRNAs, including lncRNA DANCR and IncRNA MEG3, were reported to be dysregulated in patients with PMOP and to be associated with disease progression $(11,12)$. Although the majority of previous studies have focused on the function of specific lncRNAs, aberrations in lncRNA expression profiles associated with the pathogenesis of PMOP have been rarely investigated. In a previous study, the IncRNA expression profiles in PMOP were assessed using RNA sequencing and a total of 51 dysregulated lncRNAs were identified in patients with PMOP (13). However, the sample size of the study was relatively small (3 PMOP and 2 control samples), suggesting that the results were sensitive to noise and had low statistical power. Therefore, the lncRNA expression profiles in PMOP should be further investigated. The present study aimed to explore the implication of aberrant lncRNAs in the pathogenesis of PMOP by analyzing expression profiles.

\section{Materials and methods}

Patients. A total of 10 patients with PMOP who were treated at the Union Hospital (Tongji Medical College, Huazhong University of Science and Technology, Wuhan, China) between January and May 2018 were enrolled in the present study. The inclusion criteria were as follows: i) Post-menopausal females; 
and ii) diagnosis of osteoporosis according to the World Health Organization (WHO) criteria (14). The diagnostic cut-off for osteoporosis, based on WHO guidelines, is defined as the bone mineral density (BMD) T-score being 2.5 standard deviations (SD) below the average value of a young adult, at the femoral neck, total hip or lumbar spine (L1-L4). The exclusion criteria were as follows: i) History of diseases affecting bone metabolism, including osteomalacia, hyperparathyroidism, hyperthyroidism, hypothyroidism, rheumatoid arthritis, hypercortisolism, chronic renal insufficiency and cancer; ii) history of diabetes mellitus; and iii) previous treatment with drugs affecting bone metabolism, including glucocorticoids, estrogens and fluorides. In addition, 10 age-matched healthy females who served as the control group were enrolled in the present study. The inclusion criteria of the control group were: i) Post-menopausal females; and ii) femoral neck, total hip or spinal L1-L4 BMD T-score $\geq-1.0$ SD. The exclusion criteria were as follows: i) History of diseases affecting bone metabolism, including osteomalacia, hyperparathyroidism, hyperthyroidism, hypothyroidism, rheumatoid arthritis, hypercortisolism, chronic renal insufficiency and cancer; ii) history of diabetes mellitus; and iii) previous treatment with drugs affecting bone metabolism, including glucocorticoids, estrogens and fluorides.

Samples. Peripheral whole blood samples were collected from all subjects and the peripheral blood mononuclear cells (PBMCs) were isolated by centrifugation according to standard methods.

RNA sequencing. Total RNA was extracted from PBMCs using TRIzol reagent (Invitrogen; Thermo Fisher Scientific, Inc.). Subsequently, the concentration, purity and integrity of the isolated RNA were measured and adjusted. Ribosomal RNA (rRNA) was removed from the total RNA using a Ribo-Zero ${ }^{\mathrm{TM}}$ rRNA Removal Kit (Epicentre; Illumina, Inc.) and the rRNA-depleted RNAs were used to construct the sequencing library. The first and second strand of the complementary DNA (cDNA) were synthesized and the library fragments were purified using the AMPure XP system (Beckman Coulter, Inc.). The cDNA fragments, preferentially $150-200$ base pairs in length, were selected. The quality of the library was assessed by PCR on the Bioanalyzer 2100 system (Agilent Technologies, Inc.). The clustering of index-coded samples was subsequently performed using the HiSeq PE Cluster Kit v4 cBot and the library was sequenced on the Illumina Hiseq X10 platform (both from Illumina, Inc.). Subsequently, 150 bp paired-end reads were produced following cluster generation. Automated quality control and adapter trimming were then performed using Trim Galore, Cutadapt and FastQC software. Finally, the trimmed reads were mapped to the human genome $\mathrm{Hg} 38$ by HISAT2 using the default parameters, followed by mapping of the quality control using the RSeQC software. The read counts of lncRNAs and mRNAs were subsequently calculated using feature Counts based on the annotation file (Homo_sapiens. GRCh38.83.gtf) of the Ensembl database (http://www. ensembl.org).

Bioinformatics analysis. LncRNAs and mRNAs that were identified in $>50 \%$ of the samples (at least 10 samples) were
Table I. Characteristics of patients with PMOP and controls.

\begin{tabular}{lccr}
\hline Parameter & PMOP $(\mathrm{n}=10)$ & Controls $(\mathrm{n}=10)$ & P-value \\
\hline Age (years) & $63.3 \pm 5.2$ & $61.5 \pm 6.6$ & 0.507 \\
BMI $\left(\mathrm{kg} / \mathrm{m}^{2}\right)$ & $21.1 \pm 2.3$ & $22.0 \pm 2.5$ & 0.413 \\
BMD-T score & & & \\
Femoral neck & $-3.18 \pm 0.39$ & $0.49 \pm 0.61$ & $<0.001$ \\
Hip & $-3.03 \pm 0.46$ & $0.71 \pm 0.95$ & $<0.001$ \\
L1-L4 & $-3.26 \pm 0.42$ & $0.79 \pm 0.98$ & $<0.001$
\end{tabular}

Values are expressed as the mean \pm standard deviation. Statistical comparisons were performed using the t-test. PMOP, post-menopausal osteoporosis; BMI, body mass index; BMD, bone mineral density.

subjected to bioinformatics analysis using the $\mathrm{R}$ software (version 3.5.3; Lucent Technologies). Subsequently, the following analyses were performed: i) Principal component analysis (PCA) of lncRNA and mRNA expression profiles was performed using the Stats package. ii) Heatmap analysis of IncRNA and mRNA expression profiles was performed using the Pheatmap package. iii) Dysregulated lncRNAs and mRNAs were analyzed using the DeSeq2 package and were visualized in Volcano plots. An adjusted P-value of $<0.05$ and a fold change (FC) of $>2.0$ according to $\mid \log _{2} \mathrm{FCl}>1$, were considered to indicate a statistically and biologically significant difference, respectively. iv) Heatmap analysis of the dysregulated lncRNAs and mRNAs was performed using the Pheatmap package. v) Gene Ontology (GO) and Kyoto Encyclopedia of Genes and Genomes (KEGG) enrichment analyses of the dysregulated IncRNAs and mRNAs were performed using the DAVID tool. A cut-off criterion of $\mathrm{P}<0.05$ was used to indicate significant differences (significant enrichment by the differentially expressed RNAs, or the co-expressed genes of the core/hub RNAs identified) for the GO terms and KEGG pathways. The top 30 significantly enriched GO terms, including the top 10 terms in the categories biological process, cellular component and molecular function, and the KEGG pathways, were determined in the present study. If $<30$ significantly enriched terms were obtained, all of them were presented. vi) The gene transcription and regulation data were visualized with a Circos plot using the RCircos package. In the present study, the cis-regulatory lncRNAs were defined as lncRNAs that acted within or overlapped with up to $300 \mathrm{~kb}$ of the mRNA gene, while the trans-regulatory lncRNAs were defined as the lncRNA molecules that acted beyond $300 \mathrm{~kb}$ in the genomic distance. These definitions were based on the chromosomal association of the IncRNAs with the mRNA molecules. Correlation coefficients of $>0.95$ were considered to indicate a significant correlation between lncRNAs and mRNAs.

\section{Results}

Characteristics. The mean age was $63.3 \pm 5.2$ and $61.5 \pm 6.6$ years and the body mass index (BMI) was $21.1 \pm 2.3$ and $22.0 \pm 2.5 \mathrm{~kg} / \mathrm{m}^{2}$ for the PMOP patients and controls, respectively (Table I). No significant differences were noted in the parameters age 
A

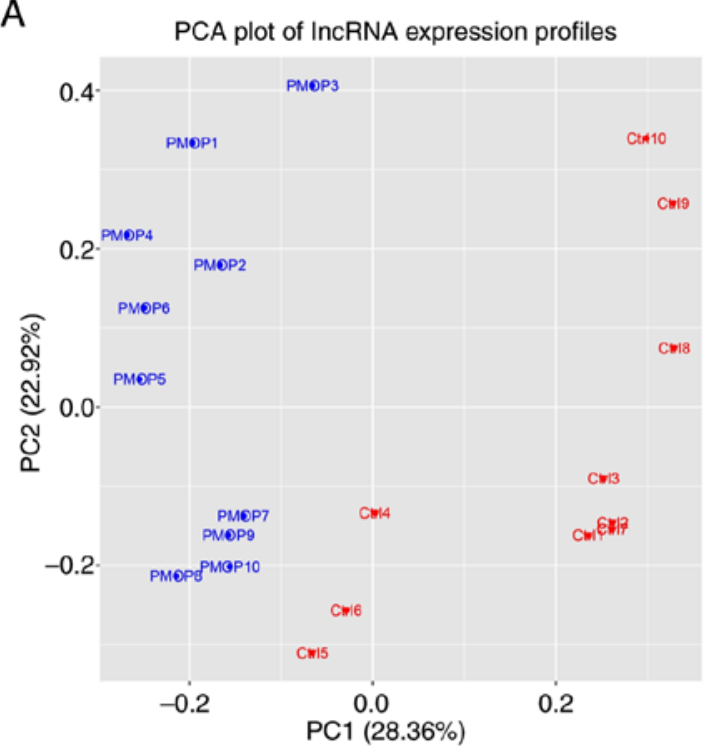

C

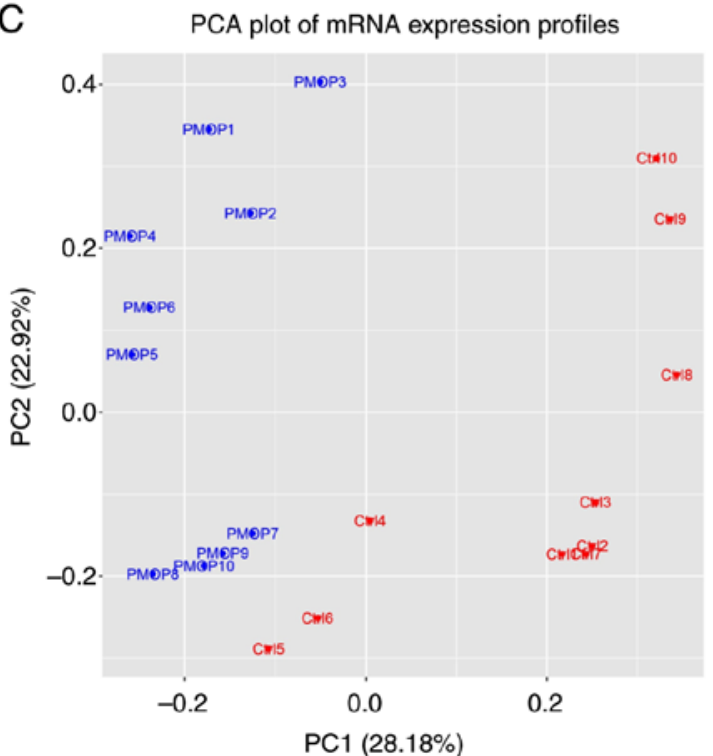

B

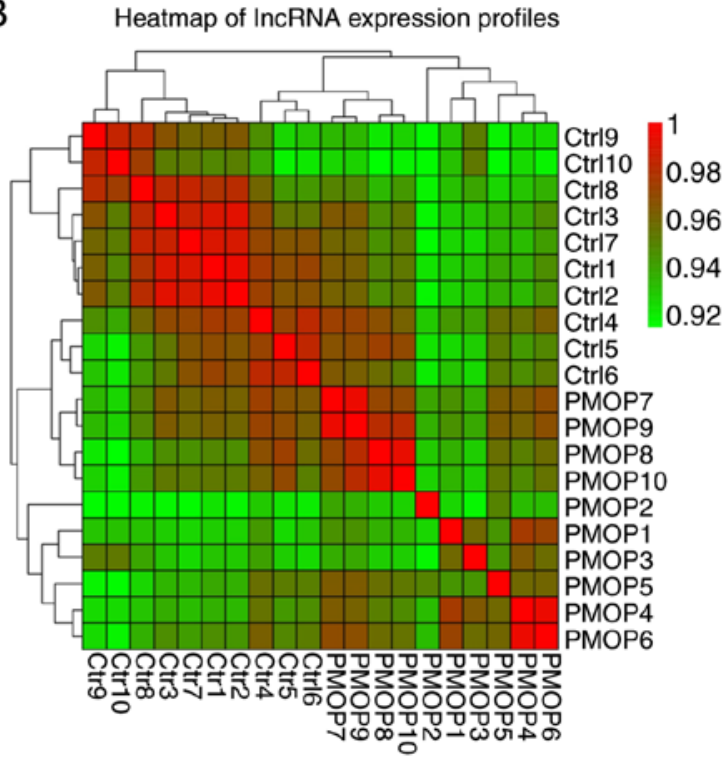

D Heatmap of mRNA expression profiles

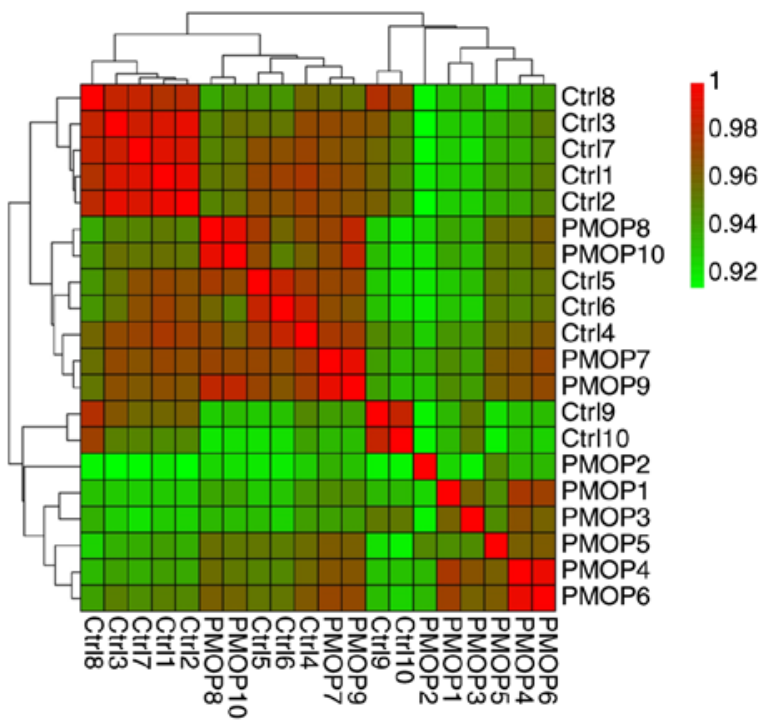

Figure 1. LncRNA and mRNA expression profiles obtained by PCA plot and heatmap analysis. (A) PCA plot of lncRNA expression profiles. (B) Heatmap analysis of lncRNA expression profiles. (C) PCA plot analysis of the mRNA expression profiles. (D) Heatmap analysis of mRNA expression profiles. PCA analysis was performed using the Stats package. Heatmap analysis was performed using the Pheatmap package. LncRNA, long non-coding RNA; PCA, principal component analysis; PMOP, post-menopausal osteoporosis; Ctrl, control.

$(\mathrm{P}=0.507)$ and $\mathrm{BMI}(\mathrm{P}=0.413)$ between $\mathrm{PMOP}$ patients and control subjects. However, increased BMD-T scores were noted in the femoral neck $(-3.18 \pm 0.39$ vs. $0.49 \pm 0.61, \mathrm{P}<0.001)$, hip $(-3.03 \pm 0.46$ vs. $0.71 \pm 0.95, \mathrm{P}<0.001)$ and lumbar spine L1-L4 $(-3.26 \pm 0.42$ vs. $0.79 \pm 0.98, \mathrm{P}<0.001)$ in $\mathrm{PMOP}$ patients compared with those in control subjects (Table I).

PCA plot and heatmap analyses of IncRNA and $m R N A$ expression profiles. Fig. 1 presents the results of the lncRNA and mRNA expression profile analysis. PCA plots are provided in Fig. $1 \mathrm{~A}$ and $\mathrm{C}$ and heatmaps are displayed in Fig. 1B and D. The data revealed differential expression profiles between PMOP patients and the control group. These results suggested that IncRNA and mRNA expression profiles may be used to discriminate PMOP patients from healthy control subjects.
Volcano plot and heatmap analyses of dysregulated lncRNAs and mRNAs. The volcano plot comprising 254 upregulated and 359 downregulated lncRNAs in patients with PMOP compared with those in the control group is provided in Fig. 2A. In the heatmap, patients with PMOP and control subjects were clustered separately based on the dysregulated expression of the lncRNAs (Fig. 2B). In addition, 522 upregulated and 1,148 downregulated mRNAs in patients with PMOP compared with those of the control group were revealed in the volcano plot (Fig. 2C), further suggesting a clustering capability of the two groups based on the expression of the dysregulated mRNAs as indicated in the heatmap (Fig. 2D).

GO and KEGG enrichment analyses for dysregulated lncRNAs and $m R N A s$. Enrichment analyses were performed to identify 

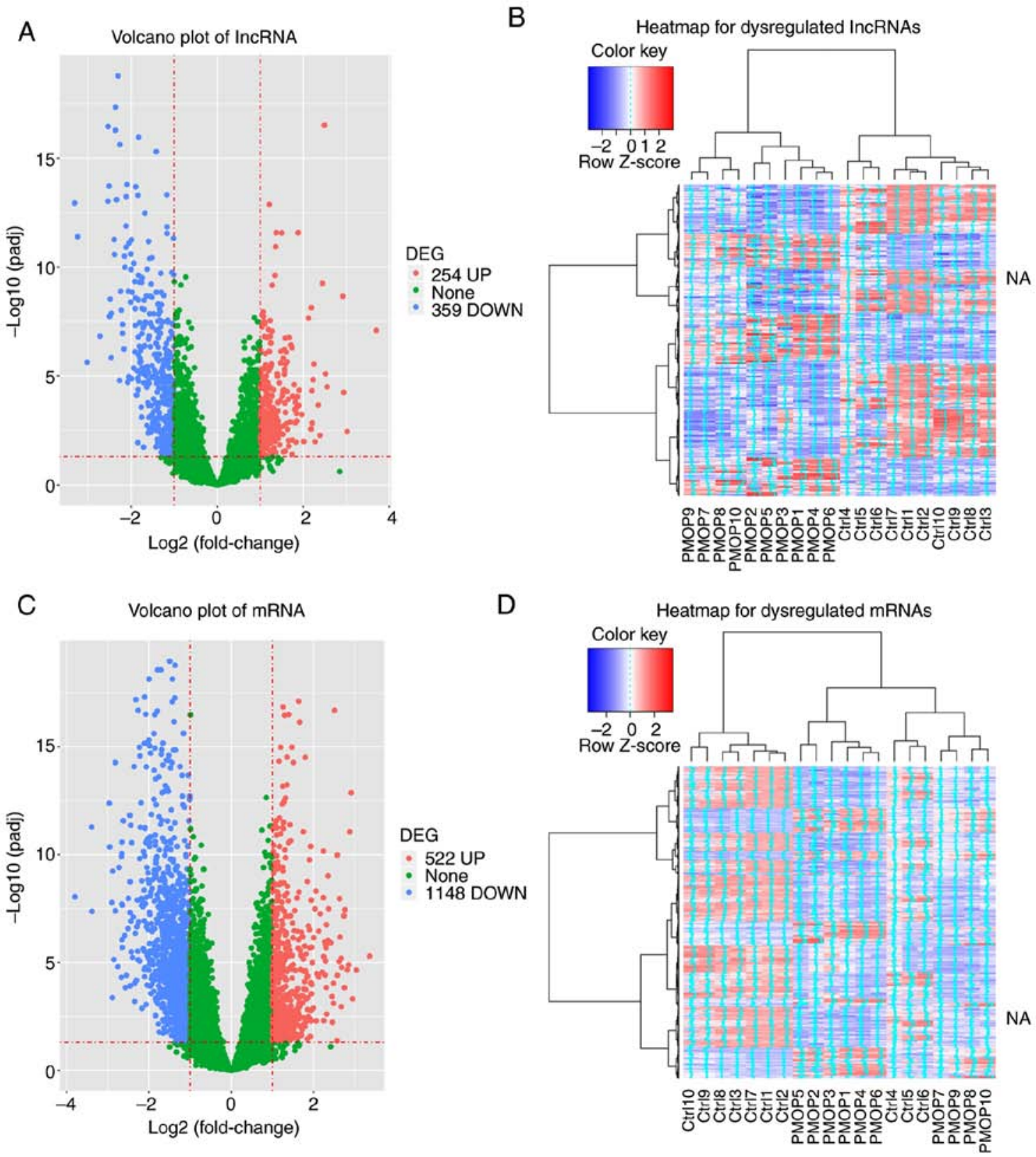

Figure 2. Identification of the dysregulated lncRNAs and mRNAs using volcano plot and heatmap analyses. (A) Volcano plot analysis of lncRNAs. (B) Heatmap analysis of dysregulated lncRNAs. (C) Volcano plot analysis of mRNAs. (D) Heatmap analysis of dysregulated mRNAs. Dysregulated lncRNAs and mRNAs were analyzed using the DeSeq2 package and visualized using Volcano spots. Statistical significance was defined as adjusted P-value $<0.05$ and biological significance was defined as $\mathrm{FC}>2.0$ according to $\log _{2} \mathrm{FCl}>1.0$. Heatmap analysis was performed using the Pheatmap package. IncRNA, long non-coding RNA; FC, fold change; Up, upregulated genes; down, downregulated genes; None, no significant change; padj, adjusted P-value; PMOP, post-menopausal osteoporosis; Ctrl, control.

the possible implications of the dysregulated lncRNAs and mRNAs in the relevant GO terms and signaling pathways. GO enrichment analysis indicated that the dysregulated lncRNAs were accumulated in various terms in the category biological process, including the apoptotic process and positive regulation of NF- $\mathrm{KB}$ signaling, as well as terms in the category cellular component, including cytosol and nucleoplasm, and terms in the category molecular function, e.g. protein binding and transcription factor activity (Fig. 3A). KEGG pathway analysis revealed that the dysregulated lncRNAs were mainly involved in PMOP-associated signaling pathways, including osteoclast differentiation and tumor necrosis factor (TNF) and mitogen-activated protein kinase (MAPK) signaling pathways (Fig. 3B). In addition, the GO enrichment analysis revealed that the dysregulated mRNAs were accumulated in terms in the category biological function, including apoptosis and protein phosphorylation, as well as terms in the category cellular component, including cytosol and membrane, and in terms in the category molecular function, e.g. metal ion and ATP binding (Fig. 3C). Finally, the KEGG pathway analysis revealed that the dysregulated mRNAs were highly associated with PMOP-associated signaling pathways in patients with diabetic complications, including osteoclast differentiation, vascular endothelial growth factor and the advanced glycation end products (AGE)/receptor for AGE (RAGE) signaling pathways (Fig. 3D). 
A

GO enrichment analysis for dysregulated IncRNAs

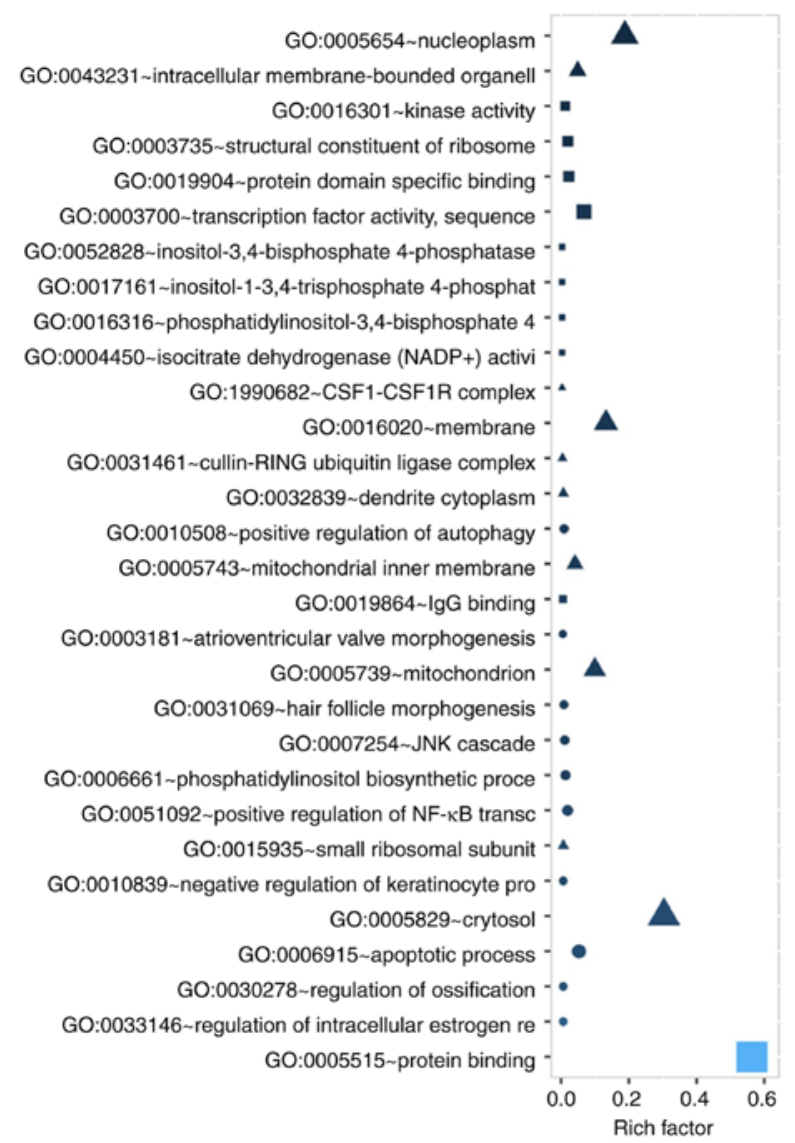

B

KEGG enrichment analysis for dysregulated IncRNAs

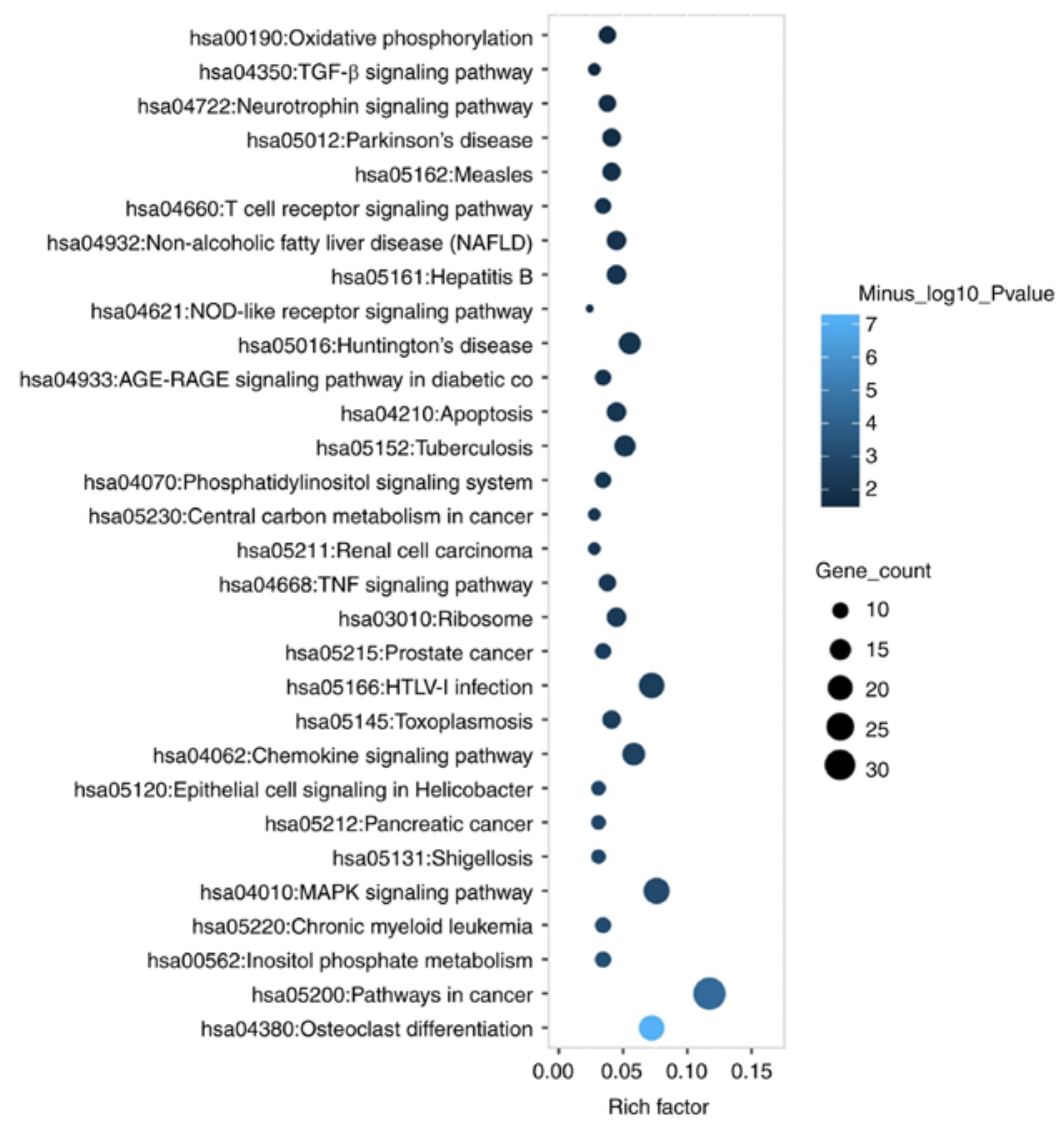

Figure 3. Functional enrichment analysis of dysregulated lncRNAs and mRNAs. (A) GO enrichment analysis of the dysregulated lncRNAs. (B) KEGG enrichment analysis of the dysregulated lncRNAs. 
GO:0003700 transcription factor activity, sequence GO:0005154 epidermal growth factor receptor bindin - " GO:0019903 protein phosphatase binding GO:0004672 protein kinase activity GO:0005524 ATP binding GO:0045121 membrane raft - 4 GO:0031996 thioesterase binding - " GO:0005884 actin filament - 1 GO:0004674 protein serine/threonine kinase activit GO:005159 insulin-like growth factor receptor bin GO:0006915 apoptotic process GO:0048013 ephrin receptor signaling pathway GO:0006468 protein phosphorylation GO:0000187 activation of MAPK activity GO:0046872 metal ion binding GO:0048010 vascular endothelial growth factor rece- • GO:0005829 cytosol GO:0016020 membrane GO:0016301 kinase activity - = GO:0071260 cellular response to mechanical stimulu GO:0030316 osteoclast differentiation - • GO:0051092 positive regulation of NF-KB transc GO:0030278 regulation of ossification - GO:0001934 positive regulation of protein phosphor -

$$
\begin{array}{llll}
0.0 & 0.1 & 0.2 & 0.3 \\
& \text { Rich factor }
\end{array}
$$

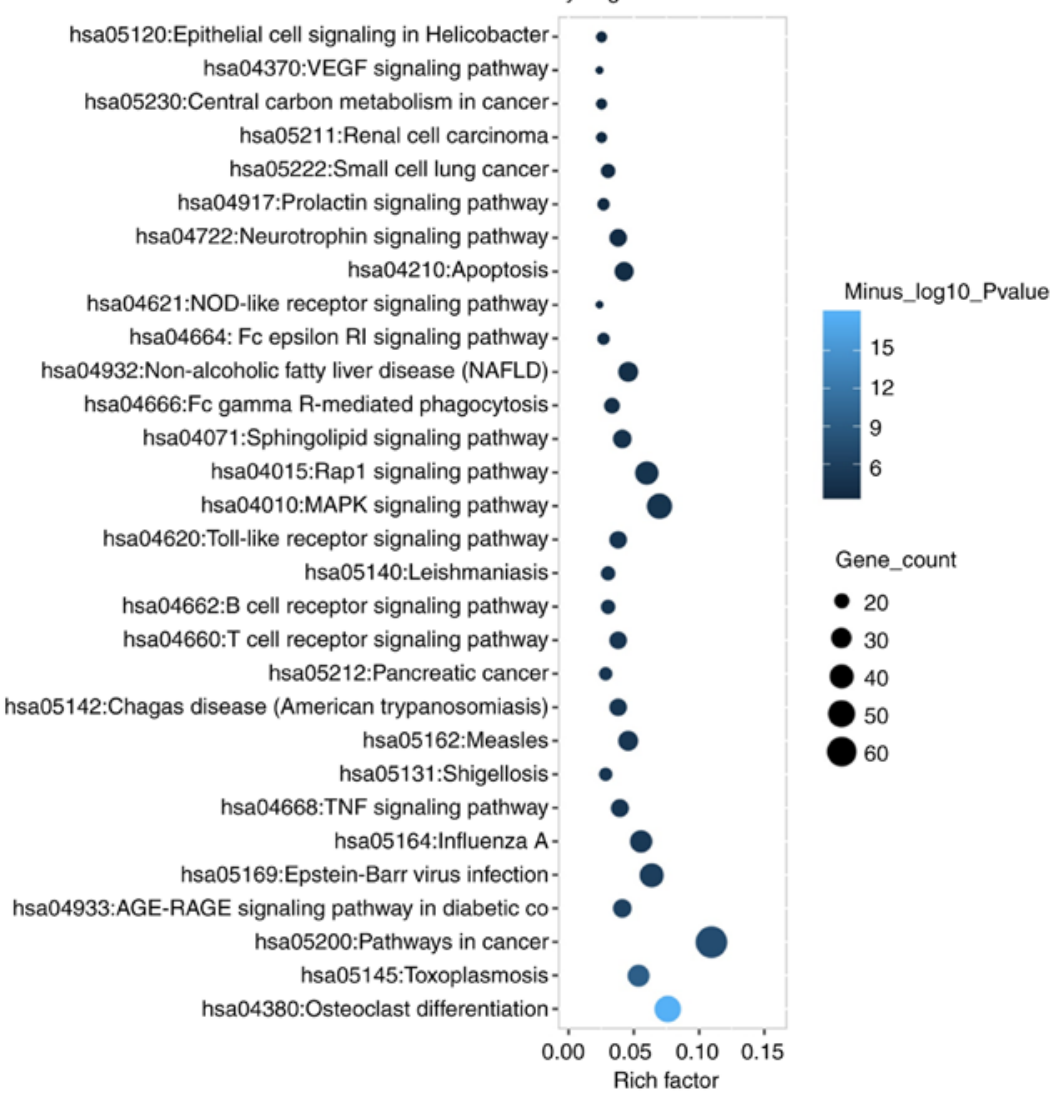

Figure 3. Continued. (C) GO enrichment analysis of the dysregulated mRNAs. (D) KEGG enrichment analysis of the dysregulated mRNAs. Enrichment analyses of dysregulated lncRNAs and mRNAs were performed using the DAVID online tool. FAT: a filter applied to remove very broad terms; BP, biological process; $\mathrm{CC}$, cellular component; MF, molecular function; TNF, tumor necrosis factor; RAGE, receptor for advanced glycation endproducts; CSF1R, colony-stimulating factor 1 receptor; TGF, transforming growth factor; VEGF, vascular endothelial growth factor; IncRNA, long non-coding RNA; GO, Gene Ontology; KEGG, Kyoto Encyclopedia of Genes and Genomes; MAPK, mitogen-activated protein kinase; hsa, Homo sapiens. 
Table II. Top 20 upregulated and 20 downregulated lncRNAs in patients with PMOP compared to controls.

A, Upregulated lncRNAs

\begin{tabular}{llcrrr}
\hline Gene symbol & \multicolumn{1}{c}{ Gene ID } & Chromosomal location & $\log _{2}$ FC & P-value \\
\hline RP11-704M14.1 & ENSG00000250696 & 4 & 3.692493 & $2.53 \times 10^{-9}$ & $8.01 \times 10^{-8}$ \\
RP11-754N21.1 & ENSG00000258084 & 12 & 3.018311 & $7.37 \times 10^{-4}$ & $3.517 \times 10^{-3}$ \\
RP11-408E5.5 & ENSG00000232243 & 13 & 2.940108 & $5.41 \times 10^{-6}$ & $5.63 \times 10^{-5}$ \\
ANKRD26P3 & ENSG00000237636 & 13 & 2.917439 & $3.72 \times 10^{-11}$ & $2.17 \times 10^{-9}$ \\
TPTEP1 & ENSG00000100181 & 22 & 2.55805 & $2.64 \times 10^{-6}$ & $3.1 \times 10^{-5}$ \\
LINC01468 & ENSG00000231131 & 10 & 2.522501 & $5.18 \times 10^{-7}$ & $7.83 \times 10^{-6}$ \\
RP11-556E13.1 & ENSG00000228651 & 10 & 2.494046 & $5.99 \times 10^{-20}$ & $3.05 \times 10^{-17}$ \\
AE000661.37 & ENSG00000251002 & 14 & 2.444645 & $8.08 \times 10^{-12}$ & $5.63 \times 10^{-10}$ \\
RP11-384P7.7 & ENSG00000260947 & 9 & 2.393043 & $1.186 \times 10^{-3}$ & $5.224 \times 10^{-3}$ \\
HMGB1P41 & ENSG00000253516 & 8 & 2.352494 & $2.63 \times 10^{-5}$ & $2.19 \times 10^{-4}$ \\
MRPL42P6 & ENSG00000242810 & 3 & 2.250902 & $1.6 \times 10^{-7}$ \\
CASC15 & ENSG00000272168 & 6 & 2.232163 & $1.08 \times 10^{-6}$ & $1.0281 \times 10^{-2}$ \\
PWAR6 & ENSG00000257151 & 15 & 2.209787 & $2.51 \times 10^{-4}$ & $1.46 \times 10^{-6}$ \\
RP11-701H24.8 & ENSG00000270246 & 15 & 2.204651 & $4.43 \times 10^{-6}$ \\
CTB-78O21.1 & ENSG00000249363 & 5 & 2.187844 & $1.47 \times 10^{-10}$ & $4.75 \times 10^{-3}$ \\
RP11-214K3.19 & ENSG00000270061 & 12 & 2.181118 & $5.27 \times 10^{-4}$ & $2.17 \times 10^{-9}$ \\
AC104088.1 & ENSG00000232555 & 2 & 2.122705 & $5.44 \times 10^{-10}$ & $2.21 \times 10^{-8}$ \\
MIR99AHG & ENSG00000215386 & 21 & 2.013722 & $2.646 \times 10^{-3}$ & $1.0099 \times 10^{-2}$ \\
RP1-34H18.1 & ENSG00000231121 & 12 & 1.964715 & $8.19 \times 10^{-5}$ & $5.71 \times 10^{-4}$ \\
LINC00890 & ENSG00000260802 & $X$ & & \\
\hline
\end{tabular}

B, Downregulated lncRNAs

\begin{tabular}{|c|c|c|c|c|c|}
\hline Gene symbol & Gene ID & Chromosomal location & $\log _{2} \mathrm{FC}$ & P-value & $\mathrm{P}_{\text {adj }}$ \\
\hline RP11-310E22.4 & ENSG00000234026 & 10 & -3.31587 & $5.97 \times 10^{-16}$ & $1.13 \times 10^{-13}$ \\
\hline RP11-326K13.4 & ENSG00000263823 & 18 & -3.24126 & $2.91 \times 10^{-14}$ & $4 \times 10^{-12}$ \\
\hline FABP5P1 & ENSG00000236972 & 13 & -3.02139 & $1.27 \times 10^{-7}$ & $2.34 \times 10^{-6}$ \\
\hline SERPINB9P1 & ENSG00000230438 & 6 & -2.72421 & $5.05 \times 10^{-9}$ & $1.49 \times 10^{-7}$ \\
\hline RPL13P2 & ENSG00000213820 & 20 & -2.53959 & $4.73 \times 10^{-16}$ & $9.27 \times 10^{-14}$ \\
\hline EEF1DP2 & ENSG00000226721 & 9 & -2.53635 & $7.67 \times 10^{-20}$ & $3.44 \times 10^{-17}$ \\
\hline RP11-529H2.2 & ENSG00000255723 & 4 & -2.51381 & $8.33 \times 10^{-17}$ & $1.91 \times 10^{-14}$ \\
\hline MIR3180-2 & ENSG00000257366 & 16 & -2.4977 & $4.34 \times 10^{-10}$ & $1.81 \times 10^{-8}$ \\
\hline LINC00672 & ENSG00000263874 & 17 & -2.48497 & $5.27 \times 10^{-11}$ & $2.95 \times 10^{-9}$ \\
\hline RP11-1148L6.9 & ENSG00000204620 & $\mathrm{X}$ & -2.46017 & $7.42 \times 10^{-8}$ & $1.47 \times 10^{-6}$ \\
\hline СТC-137K3.1 & ENSG00000270137 & 8 & -2.4554 & $2.97 \times 10^{-10}$ & $1.31 \times 10^{-8}$ \\
\hline FLJ45079 & ENSG00000204283 & 17 & -2.39574 & $4.27 \times 10^{-14}$ & $5.47 \times 10^{-12}$ \\
\hline RP11-93O17.2 & ENSG00000206356 & 5 & -2.38807 & $2.47 \times 10^{-13}$ & $2.58 \times 10^{-11}$ \\
\hline LINC01481 & ENSG00000257613 & 12 & -2.36361 & $1.17 \times 10^{-19}$ & $5.12 \times 10^{-17}$ \\
\hline NCF1B & ENSG00000182487 & 7 & -2.36149 & $6.22 \times 10^{-21}$ & $4.5 \times 10^{-18}$ \\
\hline CTD-2023M8.1 & ENSG00000248693 & 5 & -2.3384 & $3.98 \times 10^{-16}$ & $7.96 \times 10^{-14}$ \\
\hline RP11-867G23.8 & ENSG00000255468 & 11 & -2.30296 & $1.82 \times 10^{-22}$ & $1.63 \times 10^{-19}$ \\
\hline RP11-498C9.15 & ENSG00000263731 & 17 & -2.26584 & $1.19 \times 10^{-6}$ & $1.58 \times 10^{-5}$ \\
\hline CTD-3126B10.4 & ENSG00000260176 & 16 & -2.26079 & $6.1 \times 10^{-19}$ & $2.39 \times 10^{-16}$ \\
\hline CTB-35F21.1 & ENSG00000249526 & 5 & -2.24419 & $2.01 \times 10^{-11}$ & $1.29 \times 10^{-9}$ \\
\hline
\end{tabular}

Top 20 upregulated and 20 downregulated lncRNAs were selected based on the rank of absolute value of $\log _{2}$ FC. lncRNA, long non-coding RNA; PMOP, post-menopausal osteoporosis; $\mathrm{P}_{\text {adj }}$, adjusted P-value; FC, fold change.

Top 20 upregulated and 20 downregulated lncRNAs. The characteristics of the top 20 upregulated and downregulated lncRNAs in patients with PMOP are presented in Table II. The top 20 upregulated lncRNAs were as follows: RP11-704M14.1, RP11-754N21.1, RP11-408E5.5, ANKRD26P3, TPTEP1, LINC01468, RP11-556E13.1, AE000661.37, RP11-384P7.7, 


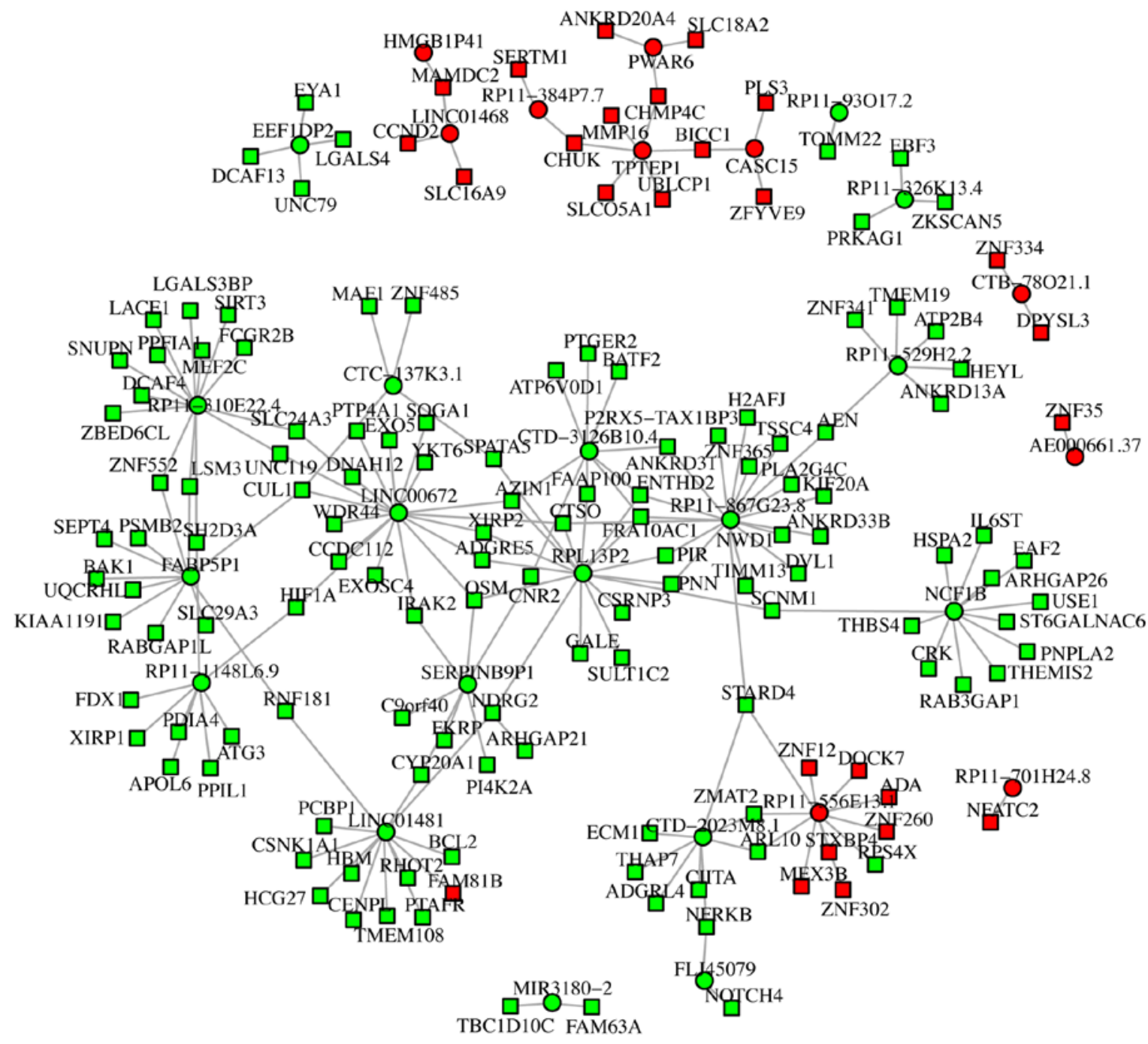

Figure 4. Regulatory network of the top dysregulated lncRNAs. The round nodes indicate the lncRNAs, and the squares indicate the mRNAs. Red symbols represent significantly upregulated and green symbols significantly downregulated lncRNAs. IncRNAs, long non-coding RNAs.

HMGB1P41, MRPL42P6, CASC15, PWAR6, RP11-701H24.8, CTB-78O21.1, RP11-214K3.19, AC104088.1, MIR99AHG, RP1-34H18.1 and LINC00890, whereas the top 20 downregulated lncRNAs were as follows: RP11-310E22.4, RP11-326K13.4, FABP5P1, SERPINB9P1, RPL13P2, EEF1DP2, RP11-529H2.2, MIR3180-2, LINC00672, RP11-1148L6.9, CTC-137K3.1, FLJ45079, RP11-93O17.2, LINC01481, NCF1B, CTD-2023M8.1, RP11-867G23.8, RP11-498C9.15, CTD-3126B10.4 and CTB-35F21.1.

Regulatory network of the top 20 up-and downregulated IncRNAs. The top 20 up- and downregulated lncRNAs were recorded. According to the target gene search criteria of the cis and trans lncRNAs, all lncRNAs identified at least one target mRNA. The regulatory network based on these results for the 10 upregulated and 18 downregulated lncRNAs is presented in Fig. 4. The regulatory network was generated and examined to further increase the understanding of the association between the dysregulated lncRNAs and target mRNAs. All mRNAs that were differentially expressed and were closely correlated with dysregulated lncRNAs were selected. A total of $25 \mathrm{lncRNAs}$ were demonstrated to directly regulate mRNAs gene expression levels. Furthermore, the majority of the lncRNAs exerted a positive regulatory effect on gene expression. In addition, among the predicted 25 lncRNAs, 13 were indicated to directly regulate the expression of $>3$ mRNAs, while the remaining 12 were demonstrated to regulate the expression of $<3$ mRNAs (Fig. 4).

Circos graph. The roles of the dysregulated lncRNAs and mRNAs in the pathology of PMOP were displayed in the Circos plots (Fig. 5). The outermost layer of the Circos plot indicates the chromosome number. The second and third outermost layers represent all differentially expressed mRNAs and lncRNAs, respectively. Finally, the inner lines represent the cis or trans lncRNA actions on the target mRNAs (Fig. 5).

\section{Discussion}

In the present study, the IncRNA expression profiles in patients with PMOP were investigated. A total of 254 upregulated and 359 downregulated IncRNAs were identified in patients with PMOP compared with those in the control group. GO enrichment analysis revealed that the dysregulated 


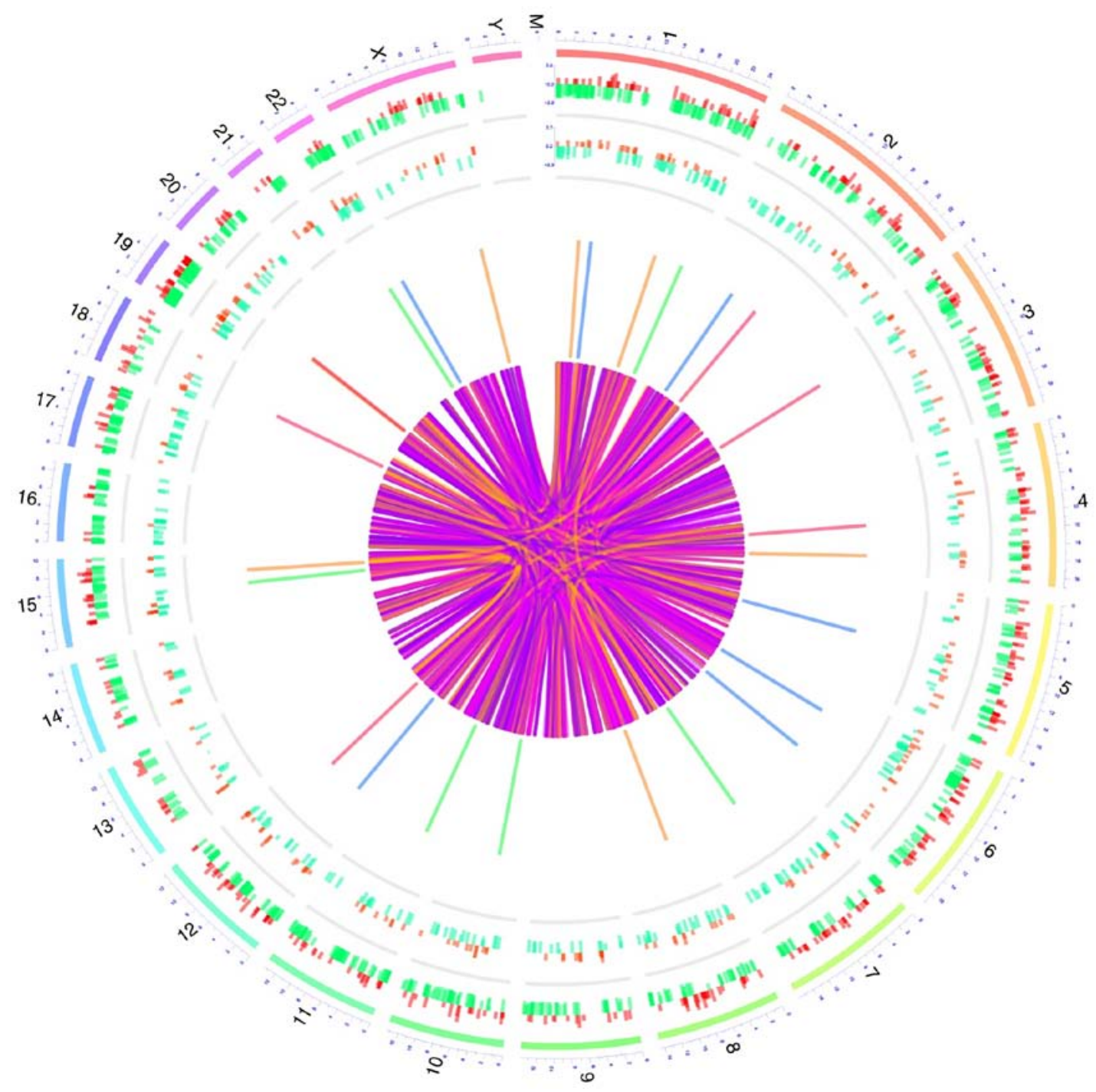

Figure 5. Visualization of transcription and regulation data using the Circos plot. The outermost layer of the Circos plot indicates the chromosome number. The second and third outermost layers represent all differentially expressed mRNAs and lncRNAs, respectively. The inner lines represent the cis or trans lncRNA actions on target mRNAs. Red color represents the upregulated genes, green color represents the downregulated genes and the height of the column represents the $\log _{2}$ (fold change). Circos plots were generated by using the RCircos package. IncRNA, long non-coding RNA.

lncRNAs were implicated in several GO terms in the category biological process, including apoptosis and positive regulation of NF- $\mathrm{KB}$ signaling. The implication of the dysregulated lncRNAs in the PMOP-associated signaling pathways was assessed using KEGG pathway analysis. The results suggested that the differentially expressed lncRNAs were accumulated in PMOP-associated signaling pathways, including osteoclast differentiation, as well as TNF and MAPK signaling pathways.

Aberrant gene expression profiles have been observed in various diseases, suggesting their potential as diagnostic biomarkers and therapeutic targets (15-17). The gene expression profiles in osteoporosis remain poorly investigated. A previous study revealed 327 upregulated and 396 downregulated genes in mesenchymal stem cells of 5 patients with osteoporosis by using microarray analysis (18). In a subsequent study, a total of 1,125 dysregulated genes (373 upregulated and 752 downregulated) were identified by microarray analysis in PBMCs derived from patients with osteoporosis (15). These results provided insight that may be used for the development of novel biomarkers and identification of treatment targets for osteoporosis.
Specific IncRNA expression profiles have been increasingly investigated in the past few years, mainly due to the implication of multiple aberrant lncRNAs in the development and progression of various diseases $(19,20)$. Recent studies revealed that aberrant lncRNA expression profiles were associated with degenerative bone diseases, including intervertebral disc degeneration (IDD) (21). The investigation of the IncRNA expression profiles in IDD using microarray technology identified 1,570 dysregulated IncRNAs in IDD patients compared with those noted in control subjects. The IncRNAs were mainly enriched in the 'extracellular matrix' and cell 'apoptosis pathways' (21). A previous study identified 1,806 IncRNAs (1,357 upregulated and 449 downregulated) and 2,307 mRNAs (1,694 upregulated and 613 downregulated) that were differentially expressed in patients with IDD compared with those in a control group (22). In addition, the levels of lncRNAs in human degenerative and normal nucleus pulposus tissues were evaluated using IncRNA-mRNA microarray. The results demonstrated that $305 \operatorname{lncRNAs}$ (135 upregulated and 170 downregulated) and 3,231 mRNAs (2,133 upregulated 
and 1,098 downregulated) were dysregulated (23). Therefore, considering the important role of lncRNA expression profiles in IDD, the r implication in the development and progression of PMOP should also be investigated. However, at present, limited information is available. A previous study identified 25 upregulated and 26 downregulated lncRNAs in total blood samples from 3 patients with PMOP compared with those in 2 healthy control subjects (13). However, further studies with a larger sample size are required to confirm the role of lncRNA expression profiles in PMOP. In the present study, PBMCs from 10 patients with PMOP and 10 healthy controls were isolated in order to investigate their corresponding lncRNA expression profiles. A total of 254 upregulated and 359 downregulated IncRNAs were identified in patients with PMOP compared with those in healthy controls. In the present study, the number of the dysregulated IncRNAs was higher compared with that in the above-mentioned study. This discrepancy may be due to various aspects. First, the sample size in the present study was larger compared with that in the previous study, which increased the statistical power of the data. In addition, deep RNA sequencing was utilized to investigate the IncRNA expression profiles in patients with PMOP. Therefore, a higher number of dysregulated lncRNAs, particularly novel lncRNAs, was identified. Finally, certain differences in the clinical features of the patients of the two studies were present. For instance, in the present study, the average age was 5 years lower compared with that in the previous study. These discrepancies may explain the increased number of dysregulated lncRNAs reported in the present study.

Functional enrichment analyses are frequently performed to reveal the potential functions of newly identified dysregulated lncRNAs in various diseases $(24,25)$. In a previous study, 25 upregulated and 26 downregulated lncRNAs were identified in patients with PMOP (13). Subsequent enrichment analyses suggested that the dysregulated lncRNAs $(n=51)$ were significantly enriched in 'membrane', 'cytosol', 'ribosome', 'cancer pathways' and in 'osteoclast differentiation'. The enrichment analysis of the present study revealed that the dysregulated lncRNAs were enriched in various GO terms in the category biological process, including 'apoptosis' and 'positive regulation of the NF- $\mathrm{NB}$ signaling pathway'. In addition, significant enrichment was noted in several signaling pathways associated with diabetic complications, including 'osteoclast differentiation' and 'TNF', 'MAPK' and 'chemokine' signaling pathway, as well as the 'AGE-RAGE signaling pathway'. These observations were consistent with previously reported data (13). The results suggested that the dysregulated lncRNAs may affect the development and progression of PMOP via the regulation of osteoclast differentiation, inflammation and apoptosis. In the present study, the regulatory network of lncRNAs and mRNAs was visualized using a Circos plot. In conclusion, the lncRNA expression profiles in patients with PMOP were investigated. In addition, novel dysregulated lncRNAs and their potential functions were identified by functional enrichment analysis. The aforementioned results may deepen the current understanding of the roles of lncRNAs in PMOP and provide an application of lncRNAs as biomarkers for PMOP and novel therapeutic targets.

The present study has certain limitations. First, the majority of the patients and healthy subjects were recruited from the Middle of China. Therefore, considering broader populations, a selection bias may have occurred. Furthermore, the expression of dysregulated lncRNAs was not verified in a larger number of patients with PMOP by in vitro detection methods, e.g. PCR. Finally, the mechanistic roles of dysregulated lncRNAs in the underlying pathogenesis of PMOP should be further investigated.

In summary, investigation of the lncRNA expression profiles in patients with PMOP and controls indicated that certain IncRNAs may be associated with the pathogenesis of PMOP via the regulation of osteoclast differentiation, inflammation and apoptosis.

\section{Acknowledgements}

Not applicable.

\section{Funding}

No funding was received.

\section{Availability of data and materials}

All data generated or analyzed during the present study are included in this published article.

\section{Author's contributions}

SW designed the experiment, performed the experiments, analyzed the data and wrote and revised the manuscript.

\section{Ethics approval and consent to participate}

Ethical approval was obtained from the Ethics Committee of the Union Hospital (Tongji Medical College, Huazhong University of Science and Technology, Wuhan, China; approval no. 2017-S927). Written informed consent was provided by all participants prior to enrollment.

\section{Patient consent for publication}

Not applicable.

\section{Competing interests}

The author declares to have no competing interests.

\section{References}

1. Ng PY, Brigitte Patricia Ribet A and Pavlos NJ: Membrane trafficking in osteoclasts and implications for osteoporosis. Biochem Soc Trans 47: 639-650, 2019.

2. Yang DH and Yang MY: The role of macrophage in the pathogenesis of osteoporosis. Int J Mol Sci 20: E2093, 2019.

3. Tanaka S: Molecular understanding of pharmacological treatment of osteoporosis. EFORT Open Rev 4: 158-164, 2019.

4. Wade SW, Strader C, Fitzpatrick LA, Anthony MS and O'Malley CD: Estimating prevalence of osteoporosis: Examples from industrialized countries. Arch Osteoporos 9: 182, 2014.

5. Shoback D, Rosen CJ, Black DM, Cheung AM, Murad MH and Eastell R: Pharmacological management of osteoporosis in postmenopausal women: An endocrine society clinical practice guideline. J Clin Endocrinol Metab 105: 2020.

6. Li N, Zheng B, Liu M, Zhou H, Zhao L, Cai H and Huang J: Cost-effectiveness of antiosteoporosis strategies for postmenopausal women with osteoporosis in China. Menopause 26: 906-914, 2019. 
7. Kanis JA, Cooper C, Rizzoli R and Reginster JY; Scientific Advisory Board of the European Society for Clinical and Economic Aspects of Osteoporosis (ESCEO) and the Committees of Scientific Advisors and National Societies of the International Osteoporosis Foundation (IOF): European guidance for the diagnosis and management of osteoporosis in postmenopausal women. Osteoporos Int 30: 3-44, 2019.

8. Dhanoa JK, Sethi RS, Verma R, Arora JS and Mukhopadhyay CS: Long non-coding RNA: Its evolutionary relics and biological implications in mammals: A review. J Anim Sci Technol 60: 25, 2018.

9. Uszczynska-Ratajczak B, Lagarde J, Frankish A, Guigó R and Johnson R: Towards a complete map of the human long non-coding RNA transcriptome. Nat Rev Genet 19: 535-548, 2018.

10. Zampetaki A, Albrecht A and Steinhofel K: Long non-coding RNA structure and function: Is there a link? Front Physiol 9: 1201,2018

11. Tong $\mathrm{X}, \mathrm{Gu} \mathrm{PC}, \mathrm{Xu} \mathrm{SZ}$ and Lin $\mathrm{XJ}$ : Long non-coding RNA-DANCR in human circulating monocytes: A potential biomarker associated with postmenopausal osteoporosis. Biosci Biotechnol Biochem 79: 732-737, 2015.

12. Wang Q, Li Y, Zhang Y, Ma L, Lin L, Meng J, Jiang L, Wang L, Zhou $\mathrm{P}$ and Zhang Y: LncRNA MEG3 inhibited osteogenic differentiation of bone marrow mesenchymal stem cells from postmenopausal osteoporosis by targeting miR-133a-3p. Biomed Pharmacother 89: 1178-1186, 2017.

13. Fei Q, Bai X, Lin J, Meng H, Yang Y and Guo A: Identification of aberrantly expressed long non-coding RNAs in postmenopausal osteoporosis. Int J Mol Med 41: 3537-3550, 2018.

14. Camacho PM, Petak SM, Binkley N, Clarke BL, Harris ST, Hurley DL, Kleerekoper M, Lewiecki EM, Miller PD, Narula HS, et al: American Association of Clinical Endocrinologists and American College of Endocrinology Clinical practice guidelines for the diagnosis and treatment of postmenopausal osteoporosis-2016. Endocr Pract 22 (Suppl 4) 1111-1118, 2016

15. Li JJ, Wang BQ, Fei Q, Yang Y and Li D: Identification of candidate genes in osteoporosis by integrated microarray analysis. Bone Joint Res 5: 594-601, 2016.

16. Chen JB, Zhu YW, Guo X, Yu C, Liu PH, Li C, Hu J, Li HH, Liu LF, Chen MF, et al: Microarray expression profiles analysis revealed IncRNA OXCT1-AS1 promoted bladder cancer cell aggressiveness via miR-455-5p/JAK1 signaling. J Cell Physiol 234: 13592-13601, 2019.
17. Wang Q,Li ZX, Li YJ,He ZG, Chen YL, Feng MH, Li SY, Wu DZ and Xiang HB: Identification of IncRNA and mRNA expression profiles in rat spinal cords at various timepoints following cardiac ischemia/reperfusion. Int J Mol Med 43: 2361-2375, 2019.

18. Zhang Y, Wang N, Ma J, Chen XC, Li Z and Zhao W: Expression profile analysis of new candidate genes for the therapy of primary osteoporosis. Eur Rev Med Pharmacol Sci 20: 433-440, 2016.

19. Luo Q, Xu C, Li X, Zeng L, Ye J, Guo Y, Huang Z and Li J: Comprehensive analysis of long non-coding RNA and mRNA expression profiles in rheumatoid arthritis. Exp Ther Med 14: 5965-5973, 2017.

20. Bountali A, Tonge DP and Mourtada-Maarabouni M: RNA sequencing reveals a key role for the long non-coding RNA MIAT in regulating neuroblastoma and glioblastoma cell fate. Int J Biol Macromol 130: 878-891, 2019.

21. Sun ZY, Yan P, Wang SJ, Liang H, Li Y, Wang DG and Tian JW: Gene expression profiles of long non-coding RNAs in human degenerated intervertebral disc tissue. Zhonghua Yi Xue Za Zhi 97: 2582-2586, 2017 (In Chinese, Abstract available in Chinese from the publisher).

22. Wan ZY, Song F, Sun Z, Chen YF, Zhang WL, Samartzis D, Ma CJ, Che L, Liu X, Ali MA, et al: Aberrantly expressed long noncoding RNAs in human intervertebral disc degeneration: A microarray related study. Arthritis Res Ther 16: 465, 2014.

23. Chen Y, Ni H, Zhao Y, Chen K, Li M, Li C, Zhu X and Fu Q: Potential role of lncRNAs in contributing to pathogenesis of intervertebral disc degeneration based on microarray data. Med Sci Monit 21: 3449-3458, 2015.

24. Nam OH, Oh TJ, Lee JH, Hwang YS and Choi SC: Differential gene expression profiles of human periodontal ligament cells preserved in Hank's balanced salt solution and milk. Dent Traumatol 36: 58-68, 2010.

25. Sun J, Wang J, Zhang N, Yang R, Chen K and Kong D: Identification of global mRNA expression profiles and comprehensive bioinformatic analyses of abnormally expressed genes in cholestatic liver disease. Gene 707: 9-21, 2019.

(i) $(-)$ This work is licensed under a Creative Commons Attribution-NonCommercial-NoDerivatives 4.0 International (CC BY-NC-ND 4.0) License. 